\title{
Controlled Synthesis of Spherical Polystyrene Beads and Their Template-Assisted Manual Assembly
}

\author{
Seo Young Yoon, Yi-Seul Park, and Jin Seok Lee* \\ Department of Chemistry, Sookmyung Women's University, Seoul 140-742, Korea. *E-mail: jinslee@sookmyung.ac.kr \\ Received February 21, 2014, Accepted April 8, 2014
}

\begin{abstract}
Polystyrene beads (PS beads) with narrow size distribution were synthesized, and their diameter was controlled from 1.2 to $5 \mu \mathrm{m}$ by varying the injection rate of a styrene solution containing initiator and the concentration of reactant, such as initiator and capping material. The diameter of the PS beads increased with increasing in the injection rates and the initiator concentration or decreasing the capping material concentration. Then, we used the PS beads as building block, and organized them into a hexagonally close-packed monolayer on substrate with template-assisted manual assembly. We showed perfect hexagonally close-packed organization of the PS beads with various sizes in large-scale area. And we demonstrated the superiority of the dry manual assembly over the wet self-assembly in terms of simplicity, speed, perfect ordering, and large scale.
\end{abstract}

Key Words : Polystyrene beads, Template-assisted manual assembly, Capping material, Initiator

\section{Introduction}

Colloidal solutions are heterogeneous mixtures in which the dispersed-phase particles are microscopically dispersed throughout another substance. They have long been used as versatile and essential building blocks of various industrial products such as foods, drinks, inks, paints, toners, coatings, papers, cosmetics, photographic films, and rheological fluids. ${ }^{1}$ In particular, spherical polymeric colloidal particles with various dimensions have attracted attention because of their potential biomedical and chromatographic applications and applications in preparing display coatings for use as coating materials and spacers in LCD displays. ${ }^{2-6}$

Efforts to synthesize colloidal particles with uniform size, shape, composition, and properties have resulted in a number of recent advances in elucidating and understanding the optical, rheological, and electrokinetic behaviors of these materials. ${ }^{7}$ Various chemical methods have now been developed to generate spherical colloids from a range of different materials such as organic polymers and inorganic compounds. ${ }^{8}$ On the basis of studies in materials science, chemistry, biology, condensed matter physics, applied optics, and fluid dynamics, ${ }^{9}$ a precise control over the properties of spherical colloids has been achieved by changing their intrinsic parameters such as the diameter, chemical composition, dispersion force, crystallinity, and surface functional group. ${ }^{10}$

The organization of these colloidal particles into twodimensional (2D) arrays on various substrates is an important area of study in modern science and technology. ${ }^{11,12}$ The ability to organize spherical colloids into spatially periodic structures has afforded interesting and often useful functionality not only from the colloidal materials but also from the long-range order exhibited by these crystalline lattices. ${ }^{13}$ As a result, the assembly of colloidal particles has attracted attention for not only fundamental research ${ }^{14}$ but also catalysis, ${ }^{15}$ chemical sensing, ${ }^{16}$ and investigations on bit-pattern- ed magnetic media. ${ }^{17-19}$ In the fabrication of photonic bandgap structures, these organizations resulted in extremely effective gravitational, convective, and electro-hydrodynamic forces. $^{20-28}$

General approaches for the organization of colloidal particles are commonly based on self-assembly processes from the solution to substrates, including sedimentation, ${ }^{29}$ evaporation, ${ }^{30}$ adsorption, ${ }^{31}$ Langmuir-Blodgett, ${ }^{32}$ and spin coating. ${ }^{33}$ Although these self-assembly methods are useful for organizing colloidal particles into hexagonally ordered arrays by using solvents, the organization of colloidal particles into large and perfect 2D arrays based on the selfassembly in solution has several drawbacks. ${ }^{20}$ For instance, defects may be easily formed on the self-assembled arrays as these arrays are fragile, only a part of the array may be organized as required, or the array formation may proceed in an uncontrollable manner. Moreover, formation of closepacked arrays over a large area is difficult, expensive and time consuming. Therefore, high-precision applications in nano-material science and engineering necessitate the organization of colloidal particles into large, perfect, defectfree arrays with precise control of array symmetry and interparticle distance, which in turn requires the development of effective and practical methods that are different from conventional methods based on self-assembly in solution.

On the basis of our recent finding that rubbing is a highly effective method for the organization of zeolite microparticles into monolayers on flat substrates, ${ }^{34}$ in this study, template with the shape of nanowell arrays was used to organize dry spherical colloidal particles into hexagonally close-packed monolayers by the manual assembly method of rubbing. For precise orientation control, polystyrene (PS) beads were synthesized with narrow size distribution as building blocks and controlled their diameter by varying the reaction temperature and the injection rate of a styrene solution containing 2,2'-azobis(2-methylpropionitrile) (AIBN) 
as the initiator. In addition, we demonstrated the superiority of the dry manual assembly over the wet self-assembly in terms of simplicity, speed, perfect ordering, and large scale.

\section{Experimental}

Chmicals. Styrene ( $\geq 99 \%$ ), 2,2'-azobis(2-methylpropionitrile) (AIBN) and polyethyleneimine (branched) (PEI) were purchsed from Sigam-Aldrich. Polyvinylpyrrolidone (K 30, average Mw 40,000) (PVP K 30) was purchased form Fluka. Silicone elastomer base and silicone elastomer curing agent (SYLGARD $\left.{ }^{\circledR} 184\right)$ were purchased from Dow-Corning. Ethyl alcohol (99.9\%), sulfuric acid and hydrogen peroxide were purchased from Duksan.

Synthesis of the Polystyrene Beads. To make the PS beads as building blocks, styrene, PVP K 30 and AIBN was used as monomer source, capping material and initiator, respectively. First, the PVP K 30 was dissolved in ethanol in three-necked round-bottom flask under argon atmosphere with stirring, then, the reaction temperature was elevated until $70{ }^{\circ} \mathrm{C}$ in which AIBN could decompose. After arrival of the reaction temperature, styrene solution containing AIBN was injected to the flask using the syringe and the injection rate was constant. Following injection, polymerization reaction was maintained for over $24 \mathrm{~h}$. The PS beads were obtained by centrifugation and washed to remove residual styrene and PVP K 30 several times. The PS beads were dried at the room temperature.

Preparation of the Patterned Si Substrates. The patterned Si wafer with hexagonal arrays with silicon pillar was fabricated by $\mathrm{KrF}$ stepping method. At first, a bottom anti-reflection (BAR) layer was spin-coated with the thickness of $58 \mathrm{~nm}$ on a 6 inch $\mathrm{Si}(100)$ wafer. And then, a positive photoresists (PR) was spin-coated onto the BARC/Si wafer. The PR/BAR/Si wafer and a photomask was placed and the set up was exposed to the KrF UV light $(248 \mathrm{~nm})$ with the non-contact and step, repeatedly. The desired pattern sizes of hole and pitch in the photomask are $3.4 \mu \mathrm{m} / 4.8 \mu \mathrm{m}, 8.5 \mu \mathrm{m} /$ $12 \mu \mathrm{m}$, and $14.2 \mu \mathrm{m} / 20 \mu \mathrm{m}$ which are four times larger than the desired image sizes on the wafer $(4 \times)$. After the $\mathrm{KrF}$ stepper, the patterned Si wafer was obtained with the pattern sizes of hole/pitch which are $0.85 \mu \mathrm{m} / 1.2 \mu \mathrm{m}, 2.125 \mu \mathrm{m} / 3$ $\mu \mathrm{m}$, and $3.55 \mu \mathrm{m} / 5 \mu \mathrm{m}$. We can employ the $1.2 \mu \mathrm{m}, 3 \mu \mathrm{m}$, and $5 \mu \mathrm{m}$, of PS beads. The irradiated PR/BAR/Si wafer was developed on a AZ 300 MIF developer. Deep etching of the PR-free $\mathrm{Si}$ areas was carried out by ion coupled plasma (ICP) using Ar-based $\mathrm{SF}_{6}$. The depths of etching were varied from $700 \mathrm{~nm}$ to $2 \mu \mathrm{m}$. The remaining BARC and PR layers after etching were removed by a PR asher. The patterned $\mathrm{Si}$ wafer was diced into $1.5 \mathrm{~cm} \times 1.5 \mathrm{~cm}$.

Assembly of the Polystyrene Beads. The glasses were coated with poly (ethyleneimine) (PEI) by spin coating. The patterned Si substrates were immersed in a piranha solution (a 3:1 mixture of sulfuric acid and hydrogen peroxide) and washed with deionized (DI) water. On the patterned Si substrates, the poly (dimethylsiloxane) (PDMS) solution which was a 10:1 mixture of silicone elastomer base and curing agent was poured and maintained in the $70{ }^{\circ} \mathrm{C}$ for few hours. When the PDMS substrate was prepared as a template, a small amount of the PS beads powder was placed on the template and this powder was rubbed using PDMS slab with uniform direction repeatedly. After rubbing process, the randomly aggregated upper layers of PS beads were removed from the bottom monolayer with hexagonally closepacking (HCP) by using a fresh sticky PDMS slab for a few seconds on top of the PS beads array and subsequently removing the PDMS slab. Finally, this monolayer of the HCP PS beads on the PDMS substrate was transferred to the pre-coated glasses with PEI.

\section{Results and Discussion}

PS beads were synthesized with various sizes by controlling the injection rate of the source which was a mixture of styrene monomer and AIBN. The size of the PS beads increased with increasing the injection rate of the source, as shown in Figure 1. As the injection rate of the source was increased from 0.1 to 0.2 , and then to $0.3 \mathrm{~mL} / \mathrm{min}$, the average particle diameters increased from 2.3 , to 3.4 , and then to $3.7 \mu \mathrm{m}$, respectively; the standard deviations for each particle size were $3.4 \%, 1.1 \%$, and $0.8 \%$, respectively. The rapid injection rate of the styrene solution resulted in the formation of larger styrene monomers for some time. PVP K 30 in the flask had a role to cap the injectioned source during the synthesis. When the injection rate of the source was fast, PVP K 30 can not embrace a large amount of source, and induced the combination of these styrene monomers with the oligomers or nanoparticles contained in the solution. This difference in the amounts of combined styrene monomers clearly explains the proportional increasing size of the PS beads under the rapid injection rate of the styrene solution.

Previous studies ${ }^{35-38}$ demonstrated that the size of PS
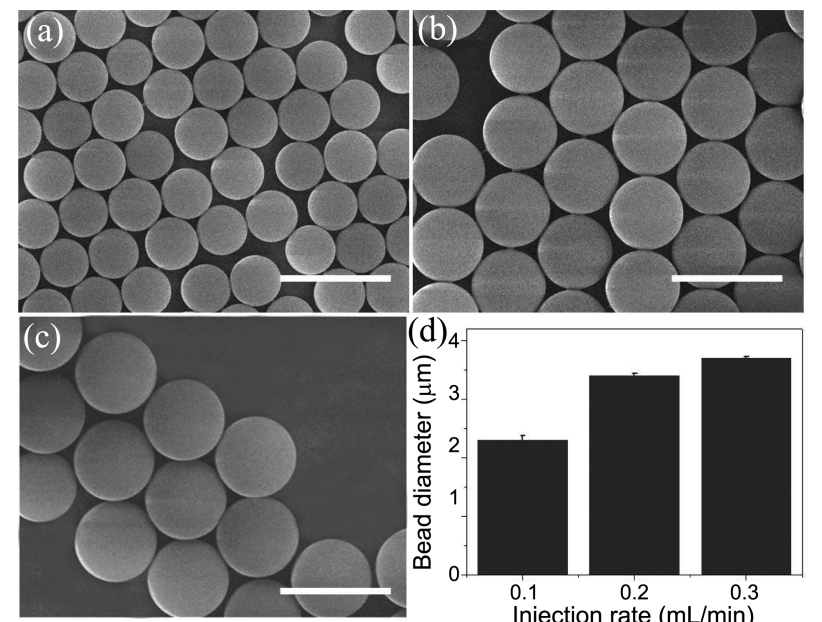

Figure 1. SEM images of the PS beads synthesized under different injection rate of the styrene solution containing AIBN: (a) 0.1, (b) 0.2 , and (c) $0.3 \mathrm{~mL} / \mathrm{min}$ at the polymerization temperature of 70 ${ }^{\circ} \mathrm{C}$. (d) The each average diameter and standard deviation of PS beads. According to an increase of the injection rate, the size of the PS beads became larger. The scale bars are $5 \mu \mathrm{m}$. 
beads is affected by not only the injection rate of the source, but also by the concentration of the initiator or capping material. With a decrease in the concentration of the initiator, AIBN, from 15.9 to $5.5 \mathrm{mM}$ under the reaction condition with $70{ }^{\circ} \mathrm{C}$ reaction temperature and $0.3 \mathrm{~mL} / \mathrm{min}$ injection rate, the particle diameter decreased from 3.7 to $1.4 \mu \mathrm{m}$, respectively, as shown in Figures 1(c) and 2(a); the corresponding particle-diameter standard deviations were $0.8 \%$ and $1.4 \%$, respectively. The dependence of the final particle size on the initiator concentration varies according to the surface charge of the initiator. ${ }^{39}$ In the case of a cationic initiator, a high initiator concentration produces many styrenefree radicals which exceed the capping ability of PVP K 30 . And then, these free radicals accelerate the growth of the existing oligomers, nuclei, and nanoparticles in the solution. Therefore, the PS particles produced at higher initiator concentrations are larger than those produced at lower initiator concentrations.

Further, the average PS bead diameter increased from 3.7 to $4.8 \mu \mathrm{m}$ as the capping material concentration was decreased from 0.31 to $0.17 \mathrm{mM}$, respectively, as shown in Figure 1(c) and 2(b); the corresponding particle-size standard deviations were $0.8 \%$, and $2.3 \%$, respectively. Because the surface charges of the PS beads and capping material were negative and positive, respectively, the PVP K 30 molecules used as the capping material could interact easily with the surface of the PS beads by ionic-dipole interactions. These surfacetethered PVP K 30 molecules prevented coagulation with the existing oligomers, nuclei, and nanoparticles in the solution. Thereby, the insufficient capping material induced the exposure of source and led to the formation of PS beads with large diameter. Therefore, the PS beads produced at lower capping material concentrations is larger than those produced at greater capping material concentrations. Through controlling the injection rate of the source and the amount of initiator and capping material, the PS beads were synthesized with various diameters.

Subsequently, the synthesized PS beads were organized into a hexagonally close-packed monolayer. ${ }^{40}$ Stepping method was applied to produce the patterned Si substrate (Figure 3(a)). Following a production of photoresist (PR)

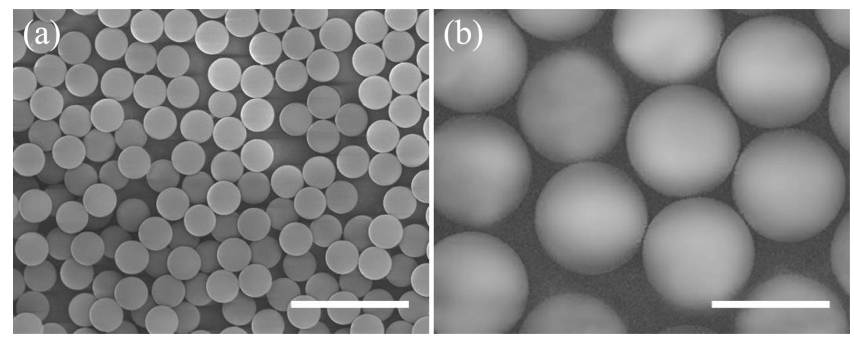

Figure 2. SEM images of the synthesized PS beads. The PS beads were synthesized in common conditions which were $70{ }^{\circ} \mathrm{C}$ of reaction temperature and $0.3 \mathrm{~mL} / \mathrm{min}$ of injection rate. (a) The size of the PS beads became smaller when the concentration of AIBN decreased from the synthesis condition (Figure 1(c)). (b) In contrast, their size became larger when the concentration of PVP K 30 decreased from the synthesis condition (Figure 1(c)). The scale bars are $5 \mu \mathrm{m}$. (a)

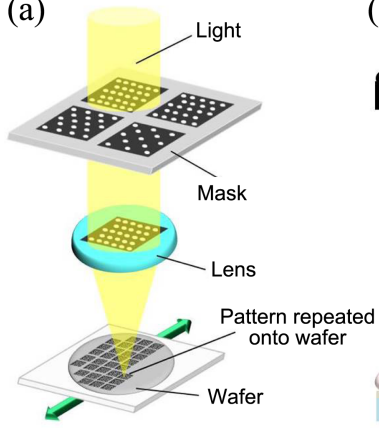

(b)

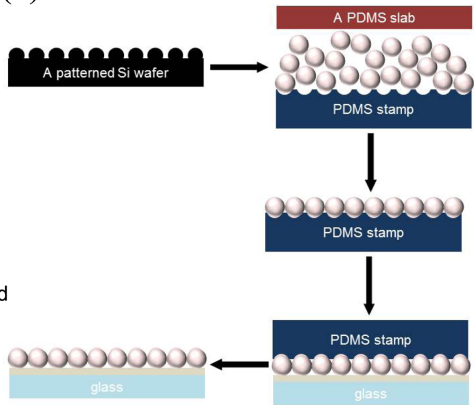

Figure 3. Schematic diagrams of the production of a patterned $\mathrm{Si}$ wafer using stepping method (a) and the general procedure to organize the PS beads into hexagonally close-packed monolayer using the template (b).

pattern on the Si wafer using mask, whose scale reduced to quarter through focusing lens. The Si wafer was etched except PR pattern on the Si wafer, and PR pattern was removed after Si etching. Then, a PDMS template with hexagonal arrays of wells was formed by pouring a PDMS solution on the patterned Si substrate (Figures 3(b)). On the PDMS template, the PS beads with different sizes from $1.2 \mu \mathrm{m}$ to 5 $\mu \mathrm{m}$ were organized by template-assisted manual assembly, as shown in Figure 4.

In a previous report, manual assembly is a very effective method for assembling zeolite microparticles on flat substrates. ${ }^{34}$ This method does not require a procedure for drying the monolayer due to the use of a powder composed of the microparticles. In addition, cracking caused by evaporation of the solvent in the Langmuir-Blodgett method is also avoided in the manual assembly. Futhermore, the large assembly area was powful strength through template-assisted manual assembly.

This very simple and fast method is affected only by the size of the PS beads and the concentration of PEI coated on substrates. The PS beads should not be aggregated and

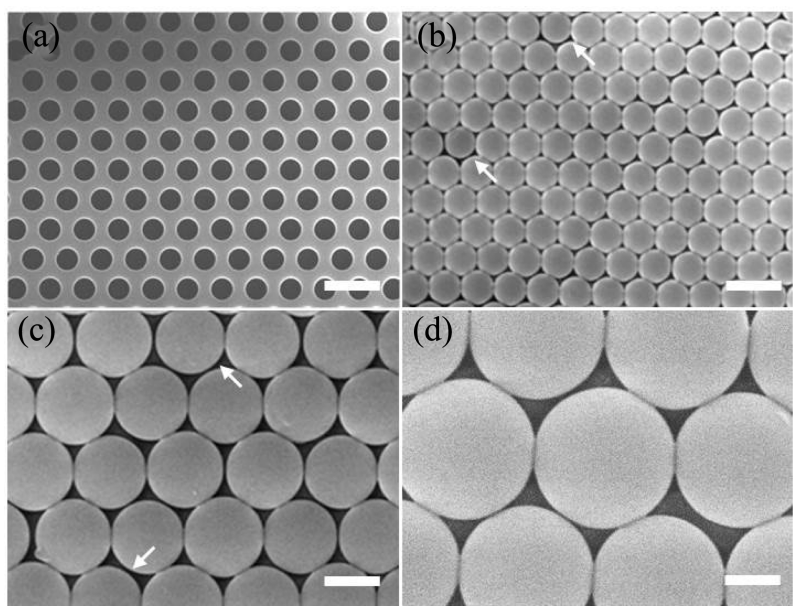

Figure 4. SEM images of the organized PS beads with different sizes. (a) The PDMS template with $1.2 \mu \mathrm{m}$ of pitch size. Each size of the PS beads is (b) 1.2 , (c) 3, and (d) $5 \mu \mathrm{m}$, respectively. The arrows indicated larger or smaller beads than the average PS bead diameter. The scale bars are $2 \mu \mathrm{m}$. 
should be uniformly sized with a narrow size distribution. The PS beads that are much larger or smaller than the average-sized PS beads could interrupt the close-packed organization of the PS beads and produce a defect of PS bead arrays. Furthermore, the size of the PS beads should be appropriate for the distance between the center of wells in the PDMS template. PS beads larger than the PDMS template, made it difficult to organize them into a perfect close-packed arrangement. On the other hand, PS beads smaller than the PDMS template were embedded into the well of template, which deformed the well of the soft and easily bendable PDMS template from spherical to oval shape and the PS bead arrays became defective (Figure 4). Therefore, the PS beads with a narrow size distribution are the most important factor.

When the PS bead monolayer was transferred from the PDMS template onto glass, the glass had to be pre-coated with PEI, which functioned as molecular glue. In comparison with zeolite microparticles, the spherical shape of the PS beads had a very small contact area with the glass substrate, weakened the binding between the beads and glass. To overcome this drawback, PEI was coated onto the glass and improved the degree of transfer. However, a high or low PEI concentration makes the transfer of PS beads difficult. Low PEI concentrations may have weakened the binding too much to enable transfer from the PDMS substrate to glass. On the other hand, high PEI concentrations may have let to PEI agglomeration, which adversely affected the hexagonally close-packed monolayer assembly of the PS beads.

\section{Conclusion}

In this work, PS beads were synthesized with different diameters ranging from 1.2 to $5 \mu \mathrm{m}$. By controlling the injection rate of the source and the concentration of the reactant, such as initiator and capping material. The size of the PS beads became larger with increasing in injection rates. In addition, their size was controlled smaller and larger by decreasing the concentration of initiator and capping material, respectively. Then, the PS beads were organized into a hexagonally close-packed monolayer by using template-assisted manual assembly, therby affording large-scale and perfect packing assembly of the PS beads. These monolayer assemblies are expected to find applications in various fields, such as in the fabrication of complex micro- and nanostructures, photonic and electronic devices, optically active materials, and bio-interfaces based on nanomaterials, which are known to influence biological responses, such as cellular adhesion, development, and proliferation.

Acknowledgments. This Research was supported by the Sookmyung Women's University Research Grants 2012.

\section{References}

1. Russel, W. B.; Saville, D. A.; Schowalter, W. R. Colloidal
Dispersions; Cambridge University Press: New York, 1989.

2. Zhang, J.; Chen, Z.; Wang, Z.; Zhang, W.; Ming, N. Mater. Lett 2003, 57, 4466.

3. Fudouz, H.; Xia, Y. Adv. Mater. 2003, 15, 892.

4. Ugelstad, J.; Stenstad, P.; Kilaas, L.; Prestvik, W. S.; Rian, A.; Nustad, K.; Herje, R.; Berge, A. Macromol. Symp. 1996, 101, 491.

5. Covolan, V. L.; Mei, L. H. I.; Rossi, C. L. Polym. Adv. Technol. 1997, 8, 44

6. Yun, D. S.; Lee, H.-S.; Jang, H. G.; Yoo, J. W. Bull. Korean Chem. Soc. 2010, 31, 1345.

7. Xia, Y.; Gates, B.; Yin, Y.; Lu, Y. Adv. Mater. 2000, 12, 693.

8. Stöber, W.; Fink, A. J. Colloid Interface Sci. 1968, 26, 62.

9. Murray, C. A.; Grier, D. G. Am. Sci. 1995, 83, 238.

10. Matijevic, E. Acc. Chem. Res. 1981, 14, 22.

11. Pileni, M. P. J. Phys. Chem. B 2001, 105, 3358.

12. McLellan, J. M.; Geissler, M.; Xia, Y. J. Am. Chem. Soc. 2004, $126,10830$.

13. Holtz, J. H.; Asher, S. A. Nature 1997, 389, 829.

14. Puntes, V. F.; Gorostiza, P.; Aruguete, D. M.; Bastus, N. G.; Alivisatos, A. P. Nat. Mater. 2004, 3, 263.

15. Lou, Y.; Maye, M. M.; Han, L.; Luo, J.; Zhong, C. J. Chem. Commun. 2001, 473.

16. Shipway, A. N.; Katz, E.; Willner, I. Chem. Phys. Chem. 2000, 1, 18.

17. Majetich, S. A.; Wen, T.; Booth, R. A. ACS Nano 2011, 5, 6081.

18. Hogg, C. R.; Majetich, S. A.; Bain, J. A. IEEE Trans. Magn. 2010, 46, 2307.

19. Sachan, M.; Bonnoit, C.; Hogg, C.; Evarts, E.; Bain, J. A.; Majetich, S. A.; Park, J. H.; Zhu, J. G. J. Phys. D: Appl. Phys. 2008, 41, 134001.

20. Ye, Y.-H.; Badilescu, S.; Truong, V.-V.; Rochon, P.; Natansohn, A. Appl. Phys. Lett. 2001, 79, 872.

21. Subramania, G.; Constant, K.; Biswas, R.; Sigalas, M.; Ho, K. Appl. Phys. Lett. 1999, 74, 3933.

22. Blanco, A.; Chomshi, E.; Grabtchak, S.; Ibisate, M.; John, S.; Leonard, S.; Lopez, C.; Meseguer, F.; Miguex, H.; Mondia, J.; Ozin, G.; Toader, O.; Driel, H. Nature (London) 2000, 405, 437.

23. Davis, K.; Russel, W.; Glantschning, W. J. Chem. Soc., Faraday Trans. 1991, 87, 411.

24. Park, S.; Qin, D.; Xia, Y. Adv. Mater. 1998, 10, 1028.

25. Denkov, N. D.; Velev, O. D.; Kralchevsky, P. A.; Ivanov, I. B.; Yoshimura, H.; Nagayama, K. Langmuir 1992, 8, 3183.

26. Jiang, P.; Bertone, J.; Hwang, K.; Colvin, V. Chem. Mater. 1999, $11,2132$.

27. Holgado, M.; Garcia-Santamaria, F.; Blanco, A.; Ibisate, M.; Cintas, A.; Miguez, H.; Serna, C.; Molpeceres, C.; Requena, J.; Mifsud, A.; Meseguer, F.; Lopez, C. Langmuir 1999, 15, 4701.

28. Jin, C.; Mclachlan, M. A.; McComb, D. W. Nano Lett. 2005, 5, 2646.

29. Micheletto, R.; Fukuda, H.; Ohtsu, M. Langmuir 1995, 11, 3333.

30. Jiang, P.; Bertone, J. F.; Hwang, K. S.; Colvin, V. L. Chem. Mater. 1999, 11, 2132.

31. Snyder, C. E.; Yake, A. M.; Feick, J. D.; Velegol, D. Langmuir $\mathbf{2 0 0 5}, 21,4813$.

32. Bardosova, M.; Pemble, M. E.; Povey, I. M.; Tredgold, R. H. $A d v$. Mater. 2010, 22, 3104.

33. Jiang, P.; McFarland, M. J. J. Am. Chem. Soc. 2004, 126, 13778.

34. Lee, J. S.; Kim, J. H.; Lee, Y. J.; Jeong, N. C.; Yoon, K. B. Angew. Chem., Int. Ed. 2007, 46, 3087.

35. Chern, C.-S.; Lin, C.-H. Polymer 2000, 41, 4473.

36. Yamamoto, T.; Iuoue, M.; Kanda, Y.; Higashitani, K. Chem. Lett. 2004, 33, 1440.

37. Paine, A. J. Colloid Interface Sci. 1990, 138, 157.

38. Im, S. H.; Khalil, G. E.; Callis, J.; Ahn, B. H.; Gouterman, M.; Xia, Y. Talanta 2005, 67, 492.

39. Yamamoto, T.; Nakayama, M.; Kanda, Y.; Higashitani, K. J. ColloidInterface Sci. 2006, 297, 112.

40. Khanh, N. N.; Yoon, K. B. J. Am. Chem. Soc. 2009, 131, 14228. 